\title{
Imported case of MERS-CoV infection identified in China, May 2015: detection and lesson learned
}

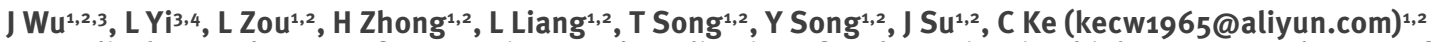

1. Medical Key Laboratory for Repository and Application of Pathogenic Microbiology, Research Center for Pathogens Detection Technology of Emerging Infectious Diseases, Guangdong Provincial Center for Disease Control and Prevention, Guangzhou, China

2. WHO Collaborating Centre for Surveillance, Research and Training of Emerging Infectious Diseases, Guangzhou, China

3. These authors contributed equally to this work

4. Guangdong Provincial Institute of Public Health, Guangzhou, China

Citation style for this article:

Wu J, Yi L, Zou L, Zhong H, Liang L, Song T, Song Y, Su J, Ke C. Imported case of MERS-CoV infection identified in China, May 2015: detection and lesson learned. Euro Surveill. 2015;20(24):pii=21158. Available online: http://www.eurosurveillance.org/ViewArticle.aspx?Articleld=21158

Article submitted on 11 June 2015 / published on 18 June 2015

At the end of May 2015, an imported case of Middle East respiratory syndrome coronavirus (MERS-CoV) infection was confirmed in China. The patient is in a stable condition and is still undergoing treatment. In this report, we summarise the preliminary findings for this imported case and the results of contact tracing. We identified 78 close contacts and after 14 days of monitoring and isolation, none of the contacts presented symptoms and all tested negative for MERS-CoV

\section{Case report}

On 27 May 2015, the Chinese Ministry of Health was notified by the World Health Organization (WHO) of a suspected case of Middle East respiratory syndrome coronavirus (MERS-CoV) infection who had travelled from South Korea to Guangdong province, China, one day earlier. The case is a man in his 40 s (Patient C) who was symptomatic during his travel but had not revealed his history of close contact with a confirmed MERS case during his stay in South Korea. The person was identified on 28 May. Medical staff wearing personal protective equipment (PPE) accompanied him to the closest hospital where he was treated in isolation in a negative pressure room. To reduce the risk of further transmission, strict infection control measures have been taken in hospital. Case investigation revealed that the man had been exposed to the first confirmed MERS case in South Korea (Patient A) who shared a ward with the father (Patient B) of Patient C. After confirmation of MERS-CoV as the cause of illness of Patient $A$ on 20 May, contact tracing confirmed MERS-CoV in Patient B on 21 May. Patient $C$ began feeling unwell on the same day because of back pain but he had no respiratory symptoms. On 25 May, his sister (Patient D) was reported to be the fourth confirmed MERS case in South Korea. On the same day, a temperature of $38.7^{\circ} \mathrm{C}$ was recorded for Patient C. The next day, on 26 May, against medical advice, the man travelled by plane from South Korea to Hong Kong directly, and then took two consecutive buses from Hong Kong airport to his destination in Guangdong province, mainland China. He stayed at Hotel A overnight and on the following day he attended meetings all day and spent the night in Hotel B until he was identified and placed in isolation. According to his quarantine form, he had fever $\left(39.7^{\circ} \mathrm{C}\right)$ but did not report his history of exposure to a MERS case upon entry into Hong Kong. Once identified and admitted to hospital on 28 May, clinical examination revealed a temperature of $39.5^{\circ} \mathrm{C}$ and non-productive cough. Over the following three days, his condition worsened. Chest x-ray showed that he had bilateral hilar infiltrates. He received ribavirin twice a day for two days, and once daily afterwards. As of 18 June, the patient remains under treatment in stable condition in hospital and would be discharged if there is no viral shedding observed for three consecutive days. The Figure illustrates the timeline of events for this case.

\section{Laboratory findings}

Diagnosis of MERS-CoV infection was performed based on the real-time RT-PCR method, using a target upstream of envelope gene (UpE) as a screening test and the open reading frame (ORF) $1 \mathrm{~b}$ gene as the confirmatory assay. MERS-CoV infection was firstly confirmed in Guangdong Centers for Disease Control (CDC) on 28 May and re-confirmed on 29 May by the national CDC in Beijing. Genome sequences were shared for assessment of possible virological changes through collaborations with the Collaborative Management Platform for detection and Analyses of (Re-) emerging and foodborne outbreaks in Europe (COMPARE) project (www.compare-europe-eu). Extensive follow-up sampling was carried out to monitor the evolution of the infection (Table).

MERS-CoV RNA was detected over eight days after fever onset, in serum samples, but only in the first four 
Timeline of events for the first imported case of MERS-CoV infection (Patient C) identified in China, May-June 2015

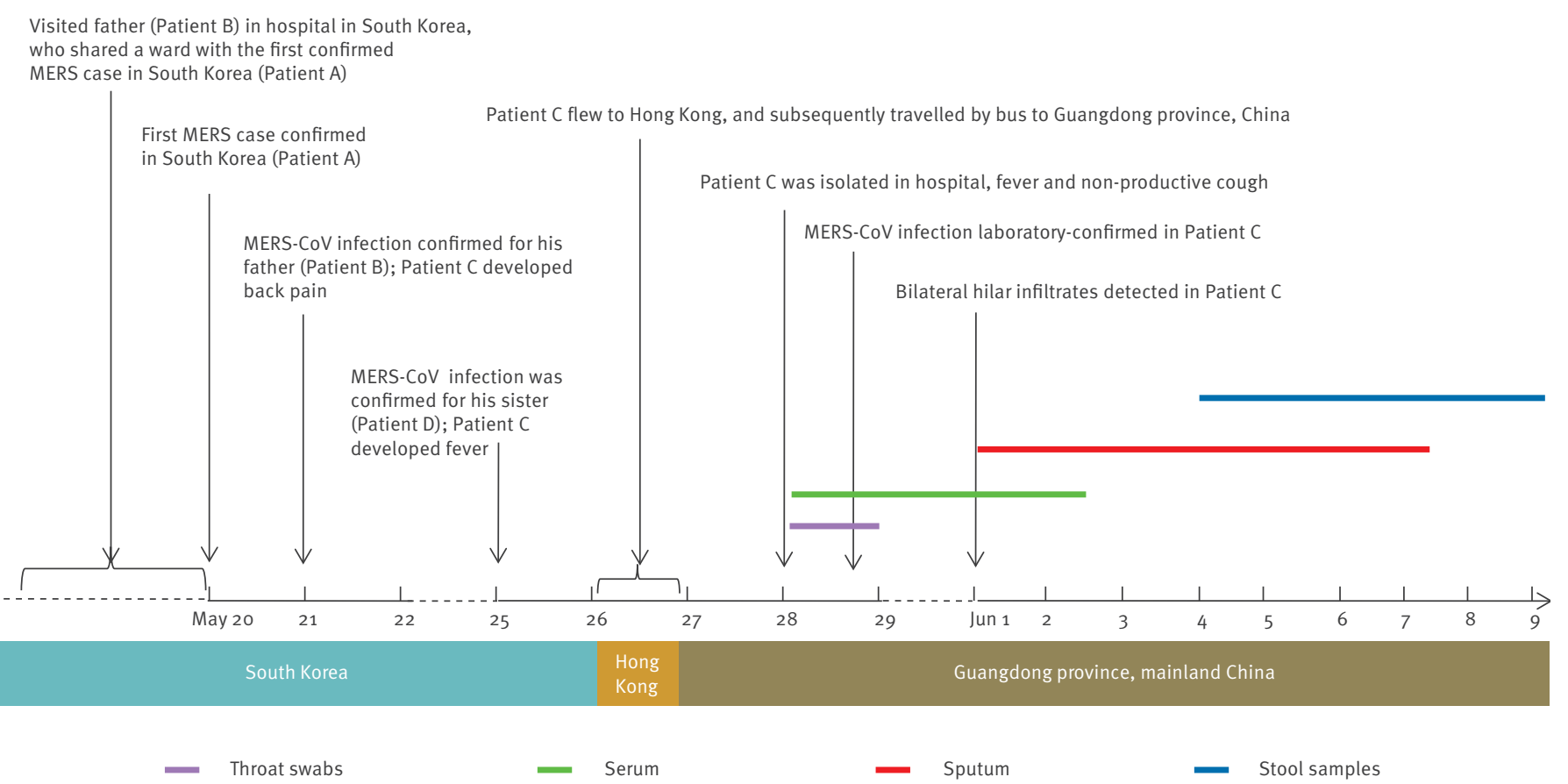

MERS-CoV: Middle East respiratory syndrome coronavirus.

The horizontal lines indicate the days when MERS-CoV was detected in various samples.

days in throat swabs. Sputum was collected and the result of the test was positive for MERS-CoV from Day 7 when pneumonia was detected. We tested two faecal samples and obtained positive results on Day 10 and 15. Sample collection and testing are still ongoing.

\section{Contact tracing}

Contact tracing was conducted immediately after the confirmation of MERS-CoV infection. According to the guidelines of the National Health and Family Planning Commission of the People's Republic of China (PRC), stewards and passengers seated two rows in front and behind the case and persons who had prolonged ( $>15$ min) face-to-face contact with the confirmed case, in any community settings (hotels, restaurants, conference rooms) were all defined as close contacts. They were included on the mandatory contact investigation list, because the case was symptomatic and potentially contagious. On 28 May, a press release was issued to inform the general public about the MERS case. Information including the travel routes of this case and preventive measures was made public via TV channels and Internet to facilitate efficient contact tracing. As of 2 June, a total of 78 close contacts including hotel staff $(n=27)$, company employees $(n=19)$, restaurant waiters $(n=13)$, bus passengers $(n=13)$, plane passengers $(n=6)$ (passengers who stayed in Hong Kong were monitored by the Hong Kong Centre for Health Protection) were identified and monitored in isolation for 14 days after their last contact with the confirmed case. After
14 days of isolation, none of the contacts presented symptoms compatible with MERS-CoV infection. Throat swabs were collected on their first and last day of quarantine and all were negative for MERS-CoV. Strict infection control measures have been taken in hospital; healthcare workers used PPE during management of this patient and therefore, they were not considered as close contacts. However, for safety reasons, serum samples were collected from 53 healthcare workers on 10 June; all were MERS-CoV negative and the follow-up is still ongoing.

\section{Discussion}

MERS-CoV is a newly emergent subgroup C betacoronavirus, with a high mortality of ca $40 \%$ [1]. As of 31 May 2015, at least 1,150 laboratory-confirmed cases including 431 related deaths have been reported to WHO [2]. Although the majority of cases occurred in countries in the Arabian Peninsula, MERS cases involving international travel occurred in at least 15 countries [3-5].

The WHO risk assessment for MERS-CoV indicated that cases will continue to be exported to other countries as a result of international travelling [2]. Here, we reported the first imported case of MERS-CoV infection identified in mainland China, related to the ongoing MERS cluster in South Korea. The first identified MERS case in South Korea was a traveler returning from the Arabian Peninsula. Unlike the situation for previously reported travel-associated MERS cases, onward transmission 
has been recently observed in South Korea, suggesting that human-to-human transmission could occur in countries outside the Arabian Peninsula and that these countries should also maintain a high level of vigilance. The WHO has published guidelines for case investigation including contact tracing since the first identification of MERS-CoV infection [6]. Control measures including quarantine of suspected cases, which have been proven effective in preventing the further spread of acute infectious diseases, may be hampered in countries with no supporting regulation in place. Therefore, in our view, without appropriate legislation, it may be more difficult to implement these recommendations. As illustrated by this incident that required a massive public health effort, infectious diseases are a global issue. While no contacts became infected in China, the spread in South Korea shows that secondary infection does constitute a risk. Therefore, until more is understood about the epidemiology and factors contributing to the spread of MERS-CoV, we believe that mandatory close monitoring and investigation of all close contacts are crucial.

This incident highlights vulnerabilities and gaps of our surveillance system, not all of which can be addressed. The early presentation of MERS-CoV or other emerging infections may not be specific [7]. Fever was observed when the case arrived in Hong Kong, but without active reporting of the previous high-risk exposure, it was reasonable not to initiate further investigation. There was no health check for this case at the entry point in mainland China, since MERS-CoV-related inquiry at

\section{TABLE}

Real-time RT-PCR results for an imported case of MERS$\mathrm{CoV}$ infection, China, May-June 2015

\begin{tabular}{|l|c|c|c|c|c|c|c|c|}
\hline \multirow{2}{*}{$\begin{array}{l}\text { Day after } \\
\text { fever } \\
\text { onset }\end{array}$} & \multicolumn{9}{|c|}{ Threshold cycle (Ct) values of MERS-CoV upE and } \\
\cline { 2 - 10 } & Throat swab & \multicolumn{2}{|c|}{ Serum } & \multicolumn{2}{c|}{ Sputum } & \multicolumn{2}{c|}{ Stool } \\
\cline { 2 - 10 } & ORF1b & upE & ORF1b & upE & ORF1b & upE & ORF1b & upE \\
\hline D3 & 32 & 32 & 36 & 39 & NA & NA & NA & NA \\
\hline D4 & 34 & 35 & 35 & 36 & NA & NA & NA & NA \\
\hline D5 & ND & ND & 34 & 32 & NA & NA & NA & NA \\
\hline D6 & ND & ND & 36 & 36 & NA & NA & NA & NA \\
\hline D7 & ND & ND & NA & NA & 34 & 36 & NA & NA \\
\hline D8 & ND & ND & 36 & 35 & 30 & 32 & NA & NA \\
\hline D9 & ND & ND & NA & NA & 29 & 31 & NA & NA \\
\hline D10 & ND & ND & ND & ND & 25 & 24 & 35 & 31 \\
\hline D11 & ND & ND & ND & ND & 25 & 26 & NA & NA \\
\hline D12 & ND & ND & ND & ND & 27 & 28 & NA & NA \\
\hline D13 & ND & ND & NA & NA & 28 & 29 & NA & NA \\
\hline D14 & NA & NA & ND & ND & NA & NA & NA & NA \\
\hline D15 & NA & NA & ND & ND & ND & ND & 36 & 36 \\
\hline & & & & & & & & \\
\hline
\end{tabular}

D: day; MERS-CoV: Middle East respiratory syndrome coronavirus; NA: not available; ND: not detected; ORF: open reading frame; upE: upstream of envelope gene.

a Day of fever onset (Do): 25 May 2015. entry point of mainland China mainly targets travellers returning from the Middle East. The increased number of countries outside the Arabian Peninsula affected by MERS-CoV highlights the need for enhanced awareness on the presence of the virus in travellers with fever from countries with ongoing epidemics. During our investigation, we observed sometimes people preferred not to disclose their history of exposure to a MERS case because of insufficient knowledge on the disease and its associated risks, or on the public health actions around it. Education of the public about MERS-CoV including symptoms, transmission modes, infection and prevention measures and risks, are critical to prevent the possible spread of MERS-CoV.

In this study, MERS-CoV RNA was detected in throat swabs only in the first two days of sampling after hospitalisation (four days after fever onset), while increased viral loads were observed in sputum seven days after fever onset when pneumonia was detected. This was consistent with previous studies that recommend that lower respiratory tract samples be given a high priority for MERS-CoV diagnosis especially in patients presenting late in their disease course with lower respiratory involvement $[8,9]$. We also obtained positive results when we tested stool and serum samples. Due to the possibility of viral shedding, comprehensive precautions for healthcare workers managing probable or confirmed MERS cases, are important. So far, data on MERS-CoV shedding were very rare and have shown different MERS-CoV detection profiles [1012]. The complete viral load profiles from a large number of patients are essential for establishing infection control measures and their necessary duration. This can also be used to monitor possible early signs of virus change: the apparent deep respiratory tract tropism of MERS-CoV in this patient was an indication that the virus causing the large cluster in South Korea did not behave differently, as concluded from the initial sequence data (data not shown). Subtle changes in the virus-host interaction that would lead to increased replication in the upper respiratory tract could potentially lead to much more efficient transmission. Therefore, detailed virological monitoring, in addition to case and contact investigations, is crucial for monitoring evolution of emerging infectious diseases.

\section{Acknowledgments}

This study was financially supported by 12 th five-year-major-projects of China's Ministry of Public Health. Grant No: 2012zX10004-213. The authors express special thanks to Marion P.G. Koopmans for her comments on the manuscript and helpful discussions.

\section{Conflict of interest}

None declared.

Authors' contributions 
All authors contributed to gathering and analysis of the information. Lina $\mathrm{Yi}$, Jie $\mathrm{Wu}$ and Changwen Ke drafted the manuscript.

\section{References}

1. Zaki AM, van Boheemen S, Bestebroer TM, Osterhaus AD, Fouchier RA. Isolation of a novel coronavirus from a man with pneumonia in Saudi Arabia. N Engl J Med. 2012;367(19):181420. http://dx.doi.org/10.1056/NEJMoa1211721 PMID:23075143

2. World Health Organization (WHO). Disease Outbreak News. Middle East respiratory syndrome coronavirus (MERS-CoV) Qatar. 31 May 2015. Geneva: WHO. Available from: http://www. who.int/csr/don/31-may-2015-mers-qatar/en/.

3. Tsiodras S, Baka A, Mentis A, Iliopoulos D, Dedoukou $X$, Papamavrou G, et al. A case of imported Middle East Respiratory Syndrome coronavirus infection and public health response, Greece, April 2014. Euro Surveill. 2014;19(16):20782. http://dx.doi.org/10.2807/1560-7917.ES2014.19.16.20782 PMID:24786258

4. Bialek SR, Allen D, Alvarado-Ramy F, Arthur R, Balajee A, Bell $D$, et al.; Centers for Disease Control and Prevention (CDC). First confirmed cases of Middle East respiratory syndrome coronavirus (MERS-CoV) infection in the United States, updated information on the epidemiology of MERS-CoV infection, and guidance for the public, clinicians, and public health authorities - May 2014. MMWR Morb Mortal Wkly Rep. 2014;63(19):431-6. PMID:24827411

5. European Centre for Disease Prevention and Control (ECDC). Severe respiratory disease associated with MERS-CoV. 5 Jun 2015. Stockholm: ECDC. 2015. Available from: http:// ecdc.europa.eu/en/publications/Publications/middle-eastrespiratory-syndrome-coronavirus-rapid-risk-assessment-5June-2015.pdf

6. World Health Organization (WHO). WHO guidelines for investigation of cases of human infection with Middle East Respiratory Syndrome Coronavirus (MERS-CoV). July 2013. Available from: http://www.who.int/csr/disease/coronavirus infections/MERS_CoV_investigation_guideline_Jul13.pdf.

7. Gańczak M. [Etiological, epidemiological and clinical aspects of coronavirus infection MERS-CoV]. [Polish]. Pol Merkur Lekarski. 2015;38(223):46-50.

8. de Sousa R, Reusken C, Koopmans M. MERS coronavirus: data gaps for laboratory preparedness. J Clin Virol. 2014;59(1):4-11. http://dx.doi.org/10.1016/j.jcv.2013.10.030 PMID:24286807

9. Drosten C, Seilmaier M, Corman VM, Hartmann W, Scheible $\mathrm{G}$, Sack S, et al. Clinical features and virological analysis of a case of Middle East respiratory syndrome coronavirus infection. Lancet Infect Dis. 2013;13(9):745-51. http://dx.doi. org/10.1016/S1473-3099(13)70154-3 PMID:23782859

10. Drosten C, Muth D, Corman VM, Hussain R, Al Masri M. HajOmar W, et al. An observational, laboratory-based study of outbreaks of middle East respiratory syndrome coronavirus in Jeddah and Riyadh, kingdom of Saudi Arabia, 2014. Clin Infect Dis. 2015;60(3):369-77.

11. Poissy J, Goffard A, Parmentier-Decrucq E, Favory R, Kauv $M$, Kipnis E, et al.; MERS-CoV Biology Group. Kinetics and pattern of viral excretion in biological specimens of two MERS-CoV cases. J Clin Virol. 2014;61(2):275-8. http://dx.doi. org/10.1016/j.jcv.2014.07.002 PMID:25073585

12. Memish ZA, Assiri AM, Al-Tawfiq JA. Middle East respiratory syndrome coronavirus (MERS-CoV) viral shedding in the respiratory tract: an observational analysis with infection control implications. Int J Infect Dis. 2014;29:307-8. http:// dx.doi.org/10.1016/j.ijid.2014.10.002 PMID:25448335 